\title{
A Retrospective Study of Micturition Interruption Exercise to Accelerate the Recovery of Urinary Incontinence after Radical Prostatectomy
}

\section{Yue Duan ( $20164919 @ z c m u . e d u . c n)$}

The Second Affiliated Hospital of Zhejiang Chinese Medical University

\section{Kangjie He}

Zhejiang Chinese Medical University

\section{Xiangnan Yang}

Zhejiang Chinese Medical University

\section{Zheng Zhu}

The Second Affiliated Hospital of Zhejiang Chinese Medical University

\section{Di Dai}

Zhejiang Chinese Medical University

Jiacheng Zhang

Zhejiang Chinese Medical University

Jiaxing Chen

Jiangshan People's Hospital

\section{Research Article}

Keywords: Kegel exercise, Micturition interruption exercise, radical prostatectomy, urinary incontinence

Posted Date: September 1st, 2021

DOl: https://doi.org/10.21203/rs.3.rs-847489/v1

License: (c) (i) This work is licensed under a Creative Commons Attribution 4.0 International License. Read Full License 


\section{Abstract}

Purpose: To explore the effectiveness of micturition interruption exercise in improving the incidence of urinary incontinence after radical prostatectomy.

Materials and Methods: With a retrospective case-control study, 96 patients admitted in the Second Affiliated Hospital of Zhejiang Chinese Medical University from August 2016 to August 2020 and underwent radical prostatectomy were collected as the subjects. Those patients who used micturition interruption exercise $(n=48)$ were set as the observation group, and the control group was collected according to the ratio of $1: 1$, the patients used Kegel exercise $(n=48)$. To compare the rehabilitation of urinary incontinence in patients and the effect of training compliance on rehabilitation.

Results: The recovery time of urinary incontinence in the observation group was significantly shorter than that of the control group. In the observation group, 83.3\% of patients with training compliance reached an average or above, while the control group only accounted for $58.3 \%$. International Consultation on Incontinence Questionnaire Short-Form score of the observation group was lower than that of the control group after surgery. Spearman analysis suggests that there is a negative correlation between the postoperative urinary incontinence recovery time and compliance with the micturition interruption exercise.

Conclusions: Micturition interruption exercise could improve the patient's exercise compliance and shorten the recovery time of urinary incontinence after radical prostatectomy.

\section{Introduction}

Radical prostatectomy (RP) is the standard treatment for localized prostate cancer, but postprostatectomy incontinence (PPI) is a tricky complication, with an incidence rate of $2-87 \%$. PPI is very common after surgery, and approximately $80 \%$ of patients have urinary incontinence one month after surgery. According to the severity of urinary incontinence, the treatment of PPI includes penis clamps, pelvic floor muscle training (PFMT), biofeedback electrical stimulation, drug therapy, and artificial urethral

sphincter, etc. ${ }^{[1,2]}$ At present, the Kegel exercise is the primary method of PFMT ${ }^{[3]}$. However, Kegel exercise has many steps, and the practice is complex, which easily leads to poor patient compliance, poor effects, and so on. "A return to continence is a major priority for the patient, the surgeon and their multidisciplinary health-care team." Sean et al. said in their article ${ }^{[4]}$. Traditional methods to improve urinary incontinence after radical prostatectomy mainly focus on technological advances in surgery and postoperative pelvic floor muscle rehabilitation.

Therefore, to optimize the effect of postoperative functional exercise, after anatomical research and clinical exploration, we simplified Kegel training and designed a set of micturition interruption exercise. To evaluate the effectiveness of this method, we collected 96 prostate cancer patients admitted to the hospital from August 2016 to August 2020. All these patients were followed up for six months by 
telephone or outpatient clinic to assess the recovery of urinary incontinence in different periods. This is reported as follows:

\section{Materials And Methods}

\subsection{Ethics}

This study was formally approved by the ethics committee of The Second Affiliated Hospital of Zhejiang Chinese Medical University (IRB No.2020-KL-167-01). Since this is a retrospective study, the ethics committee has abandoned the written notification requirement for informed consent. But we still obtained informed consent from all subjects (all patients have the ability to act independently), and they have informed and agreed to this study.All methods were carried out in accordance with relevant guidelines and regulations.

\subsection{Design}

Through the medical record system of our hospital, 96 patients who underwent radical prostatectomy were retrospectively included. The operations were performed by the same surgeon (before that, the surgeon completed approximately 50 cases of laparoscopic radical prostatectomy).

According to the different interventions they received, they were assigned to the micturition interruption exercise group and the Kegel exercise group. 48 patients received micturition interruption exercise and were assigned to observation, while another 48 patients received Kegel exercise and were assigned to the control group. Patients in both groups were followed up for at least 6 months. Follow up by telephone or urology clinic at 1,3 , and 6 months.

\subsection{Eligibility criteria}

This retrospective study was conducted in the Second Affiliated Hospital of Zhejiang University of Traditional Chinese Medicine from August 2016 to August 2020. All 96 patients were diagnosed with urinary incontinence after radical prostatectomy. Inclusion criteria include: $\geq 50$ years old, pathologically diagnosed as prostate cancer, normal cognitive function, able to complete urination interruption exercise and follow-up; moderate and above urinary incontinence was diagnosed by 1-hour urine pad test; urination function was not significantly affected before surgery, urinary incontinence occurred after RP; no pelvic surgery in the past Surgery; the patient or primary caregiver agrees to the care plan. Exclusion criteria include: previous history of prostate surgery; combined with severe heart, lung, liver, kidney, brain and other dysfunctions (expected to be unable to continue exercising).

\subsection{Interventions}


After the catheter was removed, evaluating the presence of urinary incontinence according to AUA/SUFU urinary incontinence guidelines ${ }^{[5]}$. A 1-hour urine pad test was performed by placing a weighed dry urine pad on the perineum in advance, and the patient was instructed to drink $500 \mathrm{ml}$ of boiled water within the

first 15 minutes and rest in bed ${ }^{[6]}$. In the next 30 minutes, the patient walked up and down the steps; in the last 15 minutes, the patient sat and stood 10 times, coughed 10 times, ran for 1 min, picked up five small objects on the ground, and washed hands with tap water for $1 \mathrm{~min}$. After the test, the placed urine pad was weighed, and the patient was asked to urinate and measure the urine output. The urine leakage volume $\geq 2 \mathrm{~g}$ was positive, and the urine leakage volume was $\geq 5 \mathrm{~g}$; that is, the degree of urinary incontinence was moderate or above.

48 patients in the observation group performed urination interruption exercises: during normal urination, the patients were instructed to consciously contract the perineal muscles, stop urination, and actively control urination. After the patient feels that he has stopped urinating, he urinates again and actively interrupts urination again. Repeat until urination is complete. After the patient masters the weaning exercise, it is recommended to perform this exercise when not urinating, that is, contract the abdomen, perineum, and anus at the same time, so that the perineal muscles feel sore. Another 48 patients in the control group received Kegel exercises: first lie down, relax the abdomen, chest, thighs and buttocks, feel the position of the pelvic floor muscles, then contract the pelvic floor muscles for 3 seconds, then relax for 3 seconds, and repeat 10 times. In 3 groups a day, the patients can feel the muscle contraction by putting their fingers into the rectum. The course of treatment was determined on the patient's urinary control and recovery.

\subsection{Observation indicators}

The follow-up content included the patient's Kegel exercise and micturition interruption exercise status and urination interval. The time, the number of urinations per day, and urinary incontinence status were investigated using the International Consultation on Incontinence Questionnaire Short-Form (ICIQ-SF), and the difficulties encountered by the patients in the training process were intervened and guided. In the 6th month, the patient was informed to come to the hospital for reexamination to learn more about the implementation of the micturition interruption exercise after discharge, compliance, and urinary control function recovery.

\subsection{Sample size calculation}

This study was a retrospective controlled study, and the observation index was the recovery time of urinary incontinence. According to estimates, the prevalence rate in the observation group at one month after surgery was $75 \%$, and the prevalence rate in the control group was $95 \%, a=0.05$, and the power was $80 \%$. Using SAS 9.4 software to calculate, the sample size of the observation group N1=47 cases, the sample size of the control group N2=47 cases, a total of at least 94 subjects were included ${ }^{[7]}$. The results 
of each test index were consistently significant between the two groups. Therefore, the influence of chance could be excluded, which indicates that there were indeed differences between the two groups.

\subsection{Statistical methods}

Data were processed using SPSS 25.0. Enumeration data were represented by cases(n\%); The measurement data with normal distribution is expressed as ' $x \pm s$, and the comparison between the two groups is performed by one-way analysis of variance; the measurement data with skewed distribution between the two groups are compared by the rank sum-test. A multivariate binary logistic regression model was used to analyze the influencing factors of rapid postoperative urinary incontinence recovery. correlation analysis was performed by Spearman correlation analysis. Significance was set at an alpha level of .05 , with a statistical power of .95 .

\section{Result}

\subsection{Basic information}

We summarized the general characteristics of patients in both groups. No statistical differences regarding all characteristics were detected between two groups in this study. Table 1 for details.

Table 1

Comparison of general characteristics between two groups. $\mathrm{n}(\%)$ 


\begin{tabular}{|c|c|c|c|c|}
\hline Characteristics & $\begin{array}{l}\text { Observation group } \\
\bigotimes n=54 \rrbracket\end{array}$ & $\begin{array}{l}\text { Control group } \\
(\mathrm{n}=48)\end{array}$ & Statistics & $P$ \\
\hline Age囚year & $69.70 \pm 5.24$ & $70.96 \pm 5.11$ & $F=1.43$ & 0.24 \\
\hline Educational background & & & $\underset{2}{\chi}=0.336$ & 0.85 \\
\hline Primary school and below & $24 \bigotimes 50.0 \rrbracket$ & $24(50.0)$ & & \\
\hline Junior high school & $10(20.8)$ & $12(25.0)$ & & \\
\hline High school and above & 14(29.2) & $12(25.0)$ & & \\
\hline Smoking & $24(50.0)$ & $26(54.2)$ & $x^{2}=0.17$ & 0.68 \\
\hline $\mathrm{BM} \otimes \mathrm{kg} / \mathrm{m}^{2} \square$ & $24.87 \pm 2.77$ & $24.96 \pm 1.81$ & $F=0.48$ & 0.49 \\
\hline Gleason score & & & $x^{2}=0.52$ & 0.47 \\
\hline $5 \bigotimes 7$ & $38(79.2)$ & $35(72.9)$ & & \\
\hline$\geq 8$ & 10(20.8) & $13(27.1)$ & & \\
\hline Course of disease $₫$ month $\rrbracket$ & $18.5 \pm 11.8$ & $14.7 \pm 7.3$ & $F=3.6$ & 0.061 \\
\hline $\begin{array}{l}\text { PSA level before surgery } \\
\text { 囚ng/ml区 }\end{array}$ & $24.13 \pm 17.51$ & $20.96 \pm 12.42$ & $\mathrm{H}=0.31$ & 0.58 \\
\hline Postoperative chemotherapy & $22 \rrbracket 45.8 \rrbracket$ & $18 \bowtie 37.5 \rrbracket$ & $x^{2}=0.69$ & 0.41 \\
\hline \multicolumn{5}{|c|}{ Data are present as mean \pm standard deviation or number (\%). } \\
\hline \multicolumn{5}{|c|}{$\mathrm{BMI}=$ Body mass index PSA= Prostate-specific antigen } \\
\hline
\end{tabular}

\subsection{The patient's recovery from urinary incontinence after Micturition interruption exercise after surgery}

We used 1-hour urine pad test to assess whether the patient has urinary incontinence (The specific steps were the same as above). The incidence of urinary incontinence in 96 patients when the catheter was removed was $100 \%(96 / 96)$, among which the incidence of urinary incontinence in the control and observation groups at one month after surgery was $95.8 \%(46 / 48)$ and $75.0 \%(36 / 48)$, respectively. After three months, the incidence of urinary incontinence was $75 \%(36 / 48)$ and $41.7 \%(20 / 48)$, respectively. The incidence of urinary incontinence at six months postoperatively was $33.3 \%(16 / 48)$ and $12.5 \%$ (6/48), respectively. There was a statistically significant difference in the incidence of urinary incontinence at each period after surgery $(P<0.01)$. Regardless of the observation group or the control 
group, the incidence at 1,3, and 6 months after surgery was lower than that when the catheter was removed after surgery. The incidence at three months after surgery was lower than that at one month after surgery, and the incidence at six months after surgery was lower than that at three months after surgery $(P<0.01)$. All patients had no apparent symptoms of urinary system infection during the follow-up period.

\subsection{Comparison of patients' compliance with Kegel exercise and micturition interruption exercise after surgery}

The patient's exercise completion status was recorded every day after the operation and scored one point for $1 \mathrm{~d}$ after completing the process. Finally, the patients were divided into the poor compliance group $(<25 \%)$, the general compliance group $(25 \%-75 \%)$, and the good compliance group $(>75 \%)$ according to the percentile.

After discharge from the hospital, the compliance of the observation group was significantly better than that of the control group, and the difference was statistically significant in the first and third months after surgery $(P<0.01)$. Table 2 for details.

Table 2.

Functional exercise compliance $₫ n \% \bigotimes$

\begin{tabular}{|c|c|c|c|c|c|c|c|}
\hline \multirow[t]{2}{*}{ Group } & \multicolumn{3}{|c|}{$\begin{array}{l}\text { Micturition Interruption Exercise } \\
\text { compliance }\end{array}$} & \multicolumn{3}{|c|}{ Kegel exercise compliance } & \multirow[t]{2}{*}{$P$} \\
\hline & $\begin{array}{l}\text { Poor } \\
\text { \<25\%囚 }\end{array}$ & $\begin{array}{l}\text { General } \\
\varangle 25 \%-75 \% \rrbracket\end{array}$ & $\begin{array}{l}\text { Good } \\
\bigotimes>75 \% \bigotimes\end{array}$ & $\begin{array}{l}\text { Poor } \\
\bigotimes<25 \% \bigotimes\end{array}$ & $\begin{array}{l}\text { General } \\
\text { \25\%-75\%】 }\end{array}$ & $\begin{array}{l}\text { Good } \\
\bigotimes>75 \% \rrbracket\end{array}$ & \\
\hline $\begin{array}{l}0 \text { month after } \\
\text { operation }\end{array}$ & 10 & 14 & 24 & 20 & 8 & 20 & 0.07 \\
\hline $\begin{array}{l}1 \text { month after } \\
\text { operation }\end{array}$ & 4 & 22 & 22 & 22 & 12 & 14 & $<0.01$ \\
\hline $\begin{array}{l}3 \text { months after } \\
\text { operation }\end{array}$ & 0 & 28 & 20 & 26 & 14 & 8 & $<0.01$ \\
\hline
\end{tabular}

\subsection{The scores of ICIQ-SF of the two groups of patients}


This table was used to investigate the incidence of urinary incontinence and the impact of urinary incontinence on patients. There were three scoring items: 1 . The number of urine leakages ( $0-5$ points); 2 . The amount of urine leakage (0-6 points); 3 . The impact of urine leakage on daily life (0-10 points) The total score was 0-21 points.

The mean ICIQ-SF score of the observation group was lower than that of the control group at 1, 3, and 6 months after surgery. It was statistically significant compared with "0 months after surgery" $(P<0.05)$. Table 3 for details.

Table 3

Comparison of the scores of ICIQ-SF between the two groups(compared with 0 months after surgery)

\begin{tabular}{|llllll|}
\hline Group & $\begin{array}{l}\text { Number } \\
\text { of cases }\end{array}$ & \begin{tabular}{l} 
O month after \\
operation \\
\cline { 2 - 5 }
\end{tabular} & $\begin{array}{l}1 \text { month after } \\
\text { operation }\end{array}$ & $\begin{array}{l}3 \text { months after } \\
\text { operation }\end{array}$ & $\begin{array}{l}6 \text { months after } \\
\text { operation }\end{array}$ \\
$\begin{array}{l}\text { ICIQ-SF scores } \\
\text { Observation }\end{array}$ & 24 & 9.96 & 6.72 & 4.20 & 3.03 \\
\hline $\begin{array}{l}\text { ICIQ-SF scores } \\
\text { group }\end{array}$ & 24 & 9.88 & 7.48 & 6.39 & 4.98 \\
\hline ICIQ-SF scores & ICIQ-SF scores \\
\hline ICIQ-SF= International Consultation on Incontinence Questionnaire Short-Form & \\
\hline
\end{tabular}

\subsection{Influential factors of postoperative urinary incontinence rehabilitation}

The results of multivariate binary logistic regression analysis showed that the patient's age at diagnosis $(\mathrm{OR}=5.36,95 \% \mathrm{Cl} 1.27-22.65, \mathrm{P}=0.022)$ and duration of disease $(\mathrm{OR}=46.3,95 \% \mathrm{Cl} 12.82 \bigotimes 167.72, \mathrm{P}<0.01)$ were Risk factors for urinary incontinence recovery within 6 months after laparoscopic radical prostatectomy. Education level $(\mathrm{OR}=0.325,95 \% \mathrm{Cl} 0.10 \otimes 1.03, \mathrm{P}<0.01)$, postoperative training compliance $(\mathrm{OR}=0.28,95 \% \mathrm{Cl} 0.11 \llbracket 0.77, \mathrm{P}=0.013)$ are within 6 months after radical prostatectomy Protective factors for urinary incontinence rehabilitation. There was no effect between whether there was chemotherapy after surgery and the duration of postoperative urinary incontinence $(P>0.05)$.

\subsection{Spearman correlation analysis of micturition interruption exercise compliance and urinary incontinence duration}


The correlation analysis between training compliance and urinary incontinence duration of the observation group showed that postoperative urinary incontinence duration was negatively correlated with urinary interruption training compliance ( $\mathrm{rs}=-0.42, \mathrm{P}<0.01)$. There was no statistically significant correlation between the training compliance and the duration of urinary incontinence in the control group $\triangle \mathrm{P}=0.074 \llbracket$.The relationship diagram is shown in Figure 1 and 2.

\section{Discussion}

The male urethral sphincter is composed of the proximal and distal urethral sphincters, and its integrity is closely related to male urinary control function. The distal urethral sphincter, including the external urethral sphincter (EUS), urethral prostatic membrane, pelvic floor muscles, and fascial tissues are particularly important for the maintenance of the patient's postoperative urinary control function ${ }^{[8]}$. The increase in external sphincter muscle strength and muscle fiber volume helps to improve the symptoms of PPI. The main muscle of the pelvic floor is the levator ani. The muscle and distal urethral sphincter together constitute the pelvic floor muscle structure. The exercise of the levator ani muscle can strengthen the function of pudendal nerve innervation and enhance pelvic floor myofascial and ligaments. In addition, the practice of the levator ani muscle provides structural support for the bladder and urethra. It improves the levator ani muscle exercise and tension to improve the ability of urinary control and reduce the incidence of stress urinary incontinence $e^{[9,10]}$.

Because most patients with prostate cancer are older and have limited understanding and acceptance, how to exercise and how to persist in exercise has always plagued most patients. Scholars have discovered early on that most patients may not perform normal contractions under guidance, and even a quarter of patients have worsened PPI after training ${ }^{[11]}$. Only $30 \%$ of men can perform correct pelvic floor muscle exercises after being instructed ${ }^{[12]}$. A retrospective analysis of postoperative urinary incontinence in patients with prostate cancer found that only under the guidance of a physical therapist, a standardized, reasonable, and effective PFMT can shorten the duration of urinary incontinence. However, Stafford et al. found that traditional PFMT only exercises the perianal rectal sphincter group while ignoring the damage to the urethral sphincter and bulbocavernosus muscle caused by the operation itself ${ }^{[13]}$. EUS belongs to the posterior urethral striated sphincter, which plays an important role in normal urinary control, while traditional pelvic floor muscle training is often concentrated in the anal sphincter area ${ }^{[14]}$. Some scholars believe that attention should be paid to the training method of the rhabdomyourethral sphincter to compensate for the loss of smooth muscle and striated muscle after prostatectomy ${ }^{[15]}$.

It has been reported that patient age, exercise compliance, and other factors are related to the recovery time of postoperative urinary incontinence ${ }^{[16,17]}$. Pelvic floor muscle training (PFMT) can reduce the severity and duration of early urinary incontinence after radical prostatectomy ${ }^{[18]}$. The mechanism of PFMT to promote the recovery of urinary incontinence after prostatectomy is to improve the coordination and strength of the pelvic floor muscles ${ }^{[19]}$. In 1948, Arnold Kegel first described the strengthening effect 
of Kegel exercises on the pelvic floor muscles, which prevented cystocele, rectocele, and urinary incontinence ${ }^{[20]}$. Unfortunately, Kegel exercises were subject to individual variability, and it was not easy to standardize the procedure.

In summary, we proposed micturition interruption exercise to help patients master the correct pelvic floor muscle contraction and increase the patient's subjective perception of the key muscle groups for urinary control, so that the patient can judge the correctness of the exercise by himself. Micturition interruption exercise hoped that patients could master the correct pelvic floor muscle contraction method by interrupting urine flow. At the same time, it was also convenient for patients to carry out without urinating.

Routine urine examinations during follow-up showed that there was no apparent urinary tract infection in both groups. There was no difference in compliance between the two groups of patients when the catheter was first removed $(P>0.05)$. However, the superiority of the observation group's exercise style began to manifest one month after the operation. This study found that the observation group patients had higher exercise compliance (83.3\%vs58.3\%). Analyze the reasons: simple and easy-to-learn method helps patients keep exercising. The recovery time of urinary incontinence in the observation group was significantly shorter than that of the control group (3.6 months vs5.4 months); the ICIQ-SF scores of the two groups were statistically significant at 1,3 , and 6 months significance. The above two results show that the micturition interruption exercise is more effective than Kegel exercise in improving postoperative urinary incontinence. It can produce a difference in curative effect in a short period. Micturition interruption exercise can help the patient exercise the pelvic floor muscles more correctly. Easy-to-learn and straightforward movements improve patient compliance and increase the training effect. Patients could directly observe their urinary control during training. A good training effect was also known as the motivation of the patient to persist in training.

The results of the Spearman correlation analysis of this study showed that the postoperative recovery time of urinary incontinence is highly correlated with the compliance of urinary interruption training; that is, the better the compliance, the shorter the duration of urinary incontinence(rs $=-0.42)$. But this relationship is limited to the observation group. From Fig. 2, we can know that the improvement of training compliance can not significantly shorten the duration of urinary incontinence. The reason may be related to the patient's inability to properly contract the pelvic floor muscles. This also reflects from the side that urination interruption exercises do have advantages in helping patients to properly train the pelvic floor muscles.

According to current research, the correctness of pelvic floor muscle exercise is more important than compliance in urinary incontinence rehabilitation ${ }^{[21]}$.The advantage of micturition interruption exercise is that the levator ani muscle and the external urethral sphincter are exercised through simple and easy-tounderstand urinary holding movements, which enhances the tension of the urethral fascia while maintaining proper tension of the distal urethral sphincter, thereby increasing the internal pressure always higher than the internal pressure of the bladder to achieve the purpose of control urination. Patients can directly observe the recovery of urinary control ability, which can increase confidence in the cure of urinary 
incontinence, so as to better perform subsequent functional exercises. In addition, at the beginning of exercise, micturition interruption exercise performed during urination could be carried out consciously through bladder function training, which is beneficial for establishing normal urination reflex ${ }^{[22]}$.

Since this is a retrospective study and the sample size was small, there may be a considerable risk of bias. But each observation index indicates that there was a difference between the two groups. In addition, there may be differences in the training of different individuals, and it is difficult to ensure that the training of patients after discharge from the hospital meets the expected expectations. However, we believe that the use of micturition interruption exercise improves the treatment effect of the disease. At the same time, it is convenient for patients to understand and grasp. The implementation of training is less restricted, which helps alleviate the negative impact of the tension between doctors and patients and the high cost of medical care. Reviewing previous literature reports, combined with the analysis of this article, we know that regardless of the training method, the correctness and compliance of the patient to the exercise are particularly important. Micturition interruption exercise improves the patient's target muscle exercise accuracy while also improving patient compliance with exercise after discharge from the hospital. It shortens the duration of postoperative urinary incontinence. Besides, micturition interruption exercise is not only helpful in helping patients train pelvic floor muscles to improve PPI. Still, it can also be used as an index to evaluate the efficacy of patient training, which is also an advantage that PFMT does not have.

In the future, we will also carry out more sample sizes and multi-center prospective clinical studies to further clarify the safety and feasibility of the micturition interruption exercise and benefit more patients.

\section{Conclusion}

At present, it is possible that scholars have proposed to use the method of holding urine to master the correct method of pelvic floor muscle exercise, but there are not many related literature reports. We have concluded through years of clinical practice research that micturition interruption exercise can help patients better and faster relieve the trouble of urinary incontinence after radical prostatectomy.

\section{Declarations}

\section{Consent for Publication:}

Not applicable.

\section{Availability of Data and Materials:}

All data generated or analyzed during this research period are included in published articles. Data are available on request to the authors. 


\section{Competing Interest:}

The authors declare no potential conflicts of interest with respect to the research, authorship, and publication of this article.

\section{Funding:}

Natural Science Foundation of Zhejiang Province for financial support (project number: Y20H270024).

\section{Author contributions and Acknowledgements:}

Kang-jie He carried out the questionnaire investigation, participated in the data analysis and drafted the manuscript. Xiang-nan Yang carried out the questionnaire investigation and performed the statistical analysis. Yue Duan conceived of the study, participated in its design and coordination and Obtained funding. Zheng Zhu participated in the training of nursing in this research. Di Dai and Jia-cheng Zhang checked the correctness of the data. Jia-xing Chen proposed critical revision of the manuscript for important intellectual content. All authors read and approved the final manuscript. Thanks to everyone above for their help.

\section{Consent for publication:}

Written informed consent for publication was obtained from all participants.

\section{References}

1. Anderson, C. A. et al. Conservative management for postprostatectomy urinary incontinence. Cochrane Database Syst Rev, 20 (1), CD001843 (2015).

2. Comiter, C. V. \& Dobberfuhl, A. D. The artificial urinary sphincter and male sling for postprostatectomy incontinence: Which patient should get which procedure? Investig Clin Urol, 57 (1), 3-13 (2016).

3. Huang, Y. C. \& Chang, K. V. Kegel Exercises (Treasure Island, America, 2020).

4. Mungovan, S. F. et al. Preoperative exercise interventions to optimize continence outcomes following radical prostatectomy. Nat Rev Urol, 18 (5), 259-281 (2021).

5. Sandhu, J. S. et al. Incontinence after Prostate Treatment: AUA/SUFU Guideline. J Urol, 202 (2), 369378 (2019).

6. Soto González, M. et al. Correlation between the 1-hour and 24-hour pad test in the assessment of male patients with post-prostatectomy urinary incontinence. Prog Urol, 28 (11), 536-541 (2018).

7. Shein-Chung Chow, J. et al. Sample size calculations in clinical research, third edition. Statistical Theory and Related Fields 2017;1(2):265-266. 
8. Moinzadeh, A., Shunaigat, A. N. \& Libertino, J. A. Urinary incontinence after radical retropubic prostatectomy: the outcome of a surgical technique. BJU Int, 92 (4), 355-359 (2003).

9. Bordoni, B., Sugumar, K., Leslie, S. W. \& Anatomy Abdomen and Pelvis, Pelvic Floor. Treasure Island,FL,2021.

10. Grimes, W. R. \& Stratton, M. Pelvic Floor Dysfunction. Treasure Island,FL,2021.

11. Bump, R. C., Hurt, W. G., Fantl, J. A. \& Wyman, J. F. Assessment of Kegel pelvic muscle exercise performance after brief verbal instruction. Am J Obstet Gynecol, 165 (2), 322-327 (1991).

12. Ribeiro, A. M. et al. Pelvic floor muscles after prostate radiation therapy: morpho-functional assessment by magnetic resonance imaging, surface electromyography and digital anal palpation. Int Braz J Urol, 47 (1), 120-130 (2021).

13. Stafford, R. E., Coughlin, G., Lutton, N. J. \& Hodges, P. W. Validity of Estimation of Pelvic Floor Muscle Activity from Transperineal Ultrasound Imaging in Men. PLoS One, 10 (12), e0144342 (2015).

14. Hall, L. M., Aljuraifani, R. \& Hodges, P. W. Design of programs to train pelvic floor muscles in men with urinary dysfunction: Systematic review. Neurourol Urodyn, 37 (7), 2053-2087 (2018).

15. Hodges, P. W. et al. Reconsideration of pelvic floor muscle training to prevent and treat incontinence after radical prostatectomy. Urol Oncol, 38 (5), 354-371 (2020).

16. Teber, D. et al. Is type 2 diabetes mellitus a predictive factor for incontinence after laparoscopic radical prostatectomy? A matched pair and multivariate analysis. J Urol, 183 (3), 1087-1091 (2010).

17. Latorre, G. F. S., de Fraga, R., Seleme, M. R., Mueller, C. V. \& Berghmans, B. An ideal e-health system for pelvic floor muscle training adherence: Systematic review. Neurourol Urodyn, 38 (1), 63-80 (2019).

18. Fernández, R. A. et al. Improvement of continence rate with pelvic floor muscle training postprostatectomy: a meta-analysis of randomized controlled trials. Urol Int, 94 (2), 125-132 (2015).

19. Hodges, P. W. et al. Reconsideration of pelvic floor muscle training to prevent and treat incontinence after radical prostatectomy. Urol Oncol, 38 (5), 354-371 (2020).

20. Kegel, A. H. Progressive resistance exercise in the functional restoration of the perineal muscles. $A m$ J Obstet Gynecol, 56 (2), 238-248 (1948).

21. Hall, L. M., Aljuraifani, R. \& Hodges, P. W. Design of programs to train pelvic floor muscles in men with urinary dysfunction: Systematic review. Neurourol Urodyn, 37 (7), 2053-2087 (2018).

22. Yanmei Wen, P. \& Ouyang Huafeng Yang, Shiwei Yang,Yi Song.Efficacy of pelvic floor muscle training and micturition interruption training on prevention of urinary retention after radical operation of gynecological tumor:a Meta analysis. Chinese Journal of Modern Nursing, 23 (33), 4244-4249 (2017).

\section{Figures}




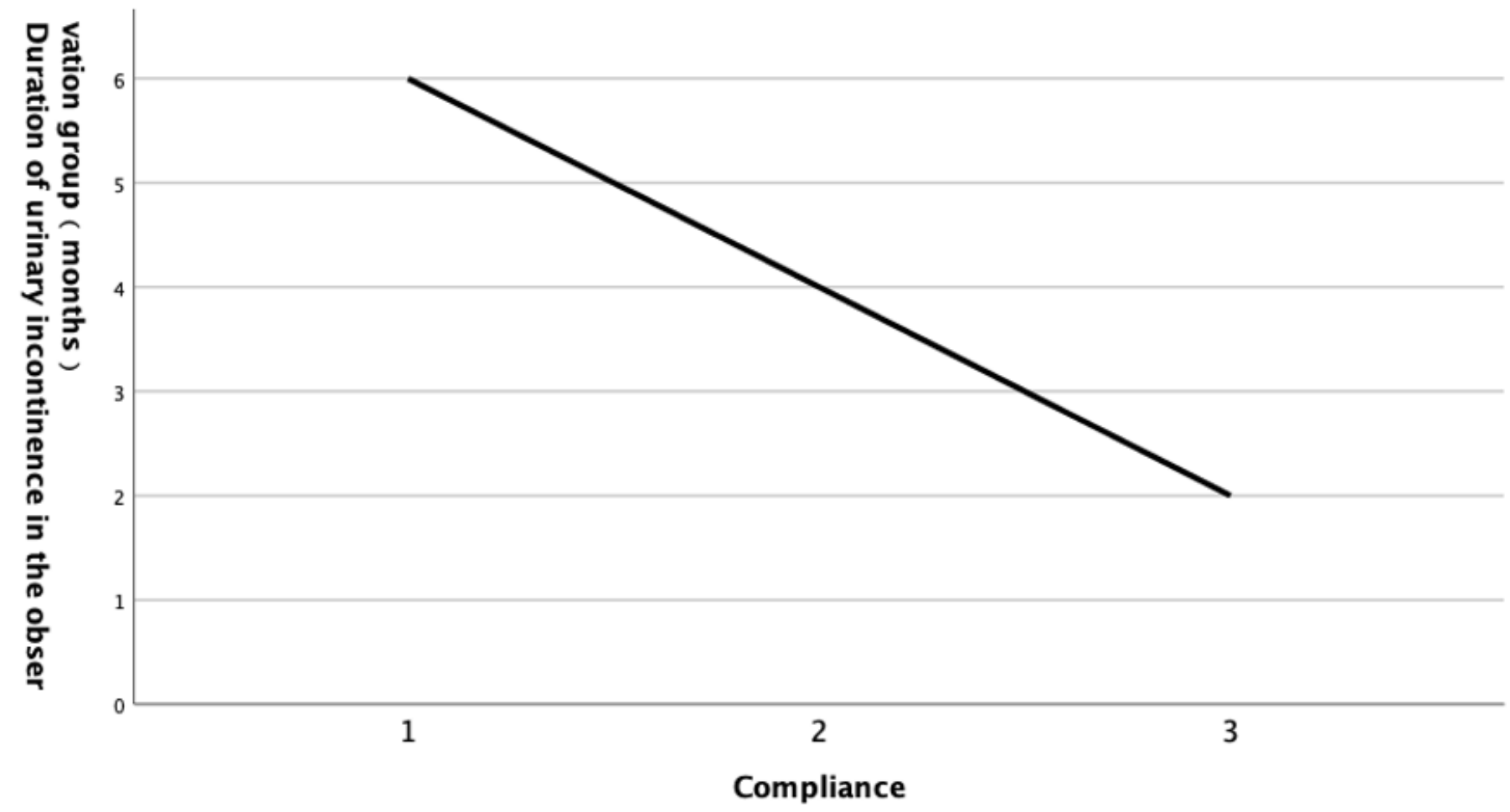

Figure 1

The relationship between the duration of urinary incontinence and exercise compliance in the observation group

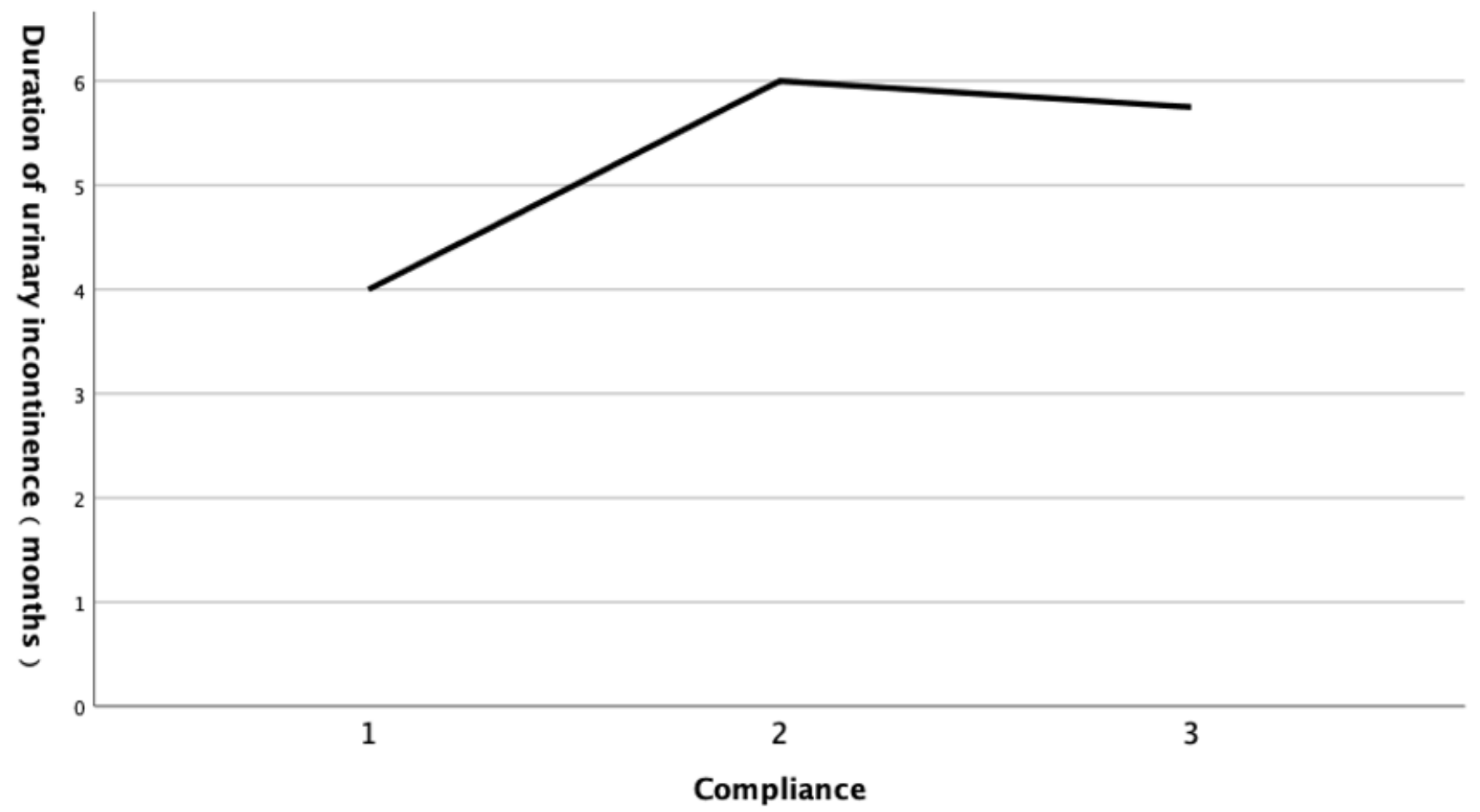

Figure 2 
The relationship between the duration of urinary incontinence and practice compliance in the control group 